\title{
LAS REPRESENTACIONES EN MATEMÁTICAS Y LA INTERVENCIÓN DEL PROFESOR: \\ UN EJEMPLO
}

\section{Luis Eduardo Espitia Supelano*}

\begin{abstract}
This article shows the teacher's role in the process needed to overcome some of the difficulties that students have, when different representations are used to illustrate the statement of a word problem.
\end{abstract}

\section{INTRODUCCIÓN}

Este escrito pretende mostrar la participación de los estudiantes y el profesor, y algunas interacciones que se efectúan en el desarrollo de una actividad simple, como es la búsqueda de una solución a un problema (?) planteado.

\section{DESARROLLO}

En un curso, para alumnos (25) de Licenciatura, de Cálculo Diferencial se propuso el siguiente problema (?): "Un rectángulo con base de longitud $X$ está inscrito en una circunferencia de radio 2. Exprese el área del rectángulo corno una función de $X$

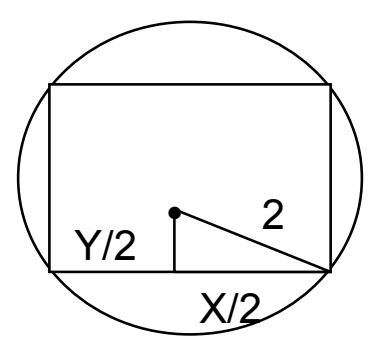

A continuación se describe la manera como se desarrolla la búsqueda de una solución.

Se plantea considerar parte del enunciado "Un rectángulo con base de longitud x está inscrito en un círculo de radio 2 ....

\footnotetext{
*Profesor Departamento de Matemáticas. Universidad Pedagógica Nacional.
} 
Como una ocurrencia espontánea (del profesor) se solicita "por favor, traducir a un gráfico el anterior enunciado". Luego de dar un tiempo prudencial se observan los siguientes gráficos:

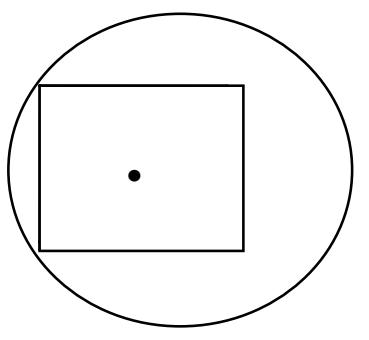

(a)

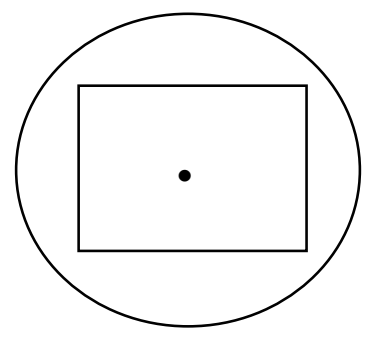

(b)

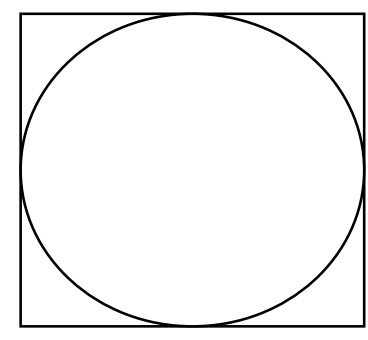

(c)

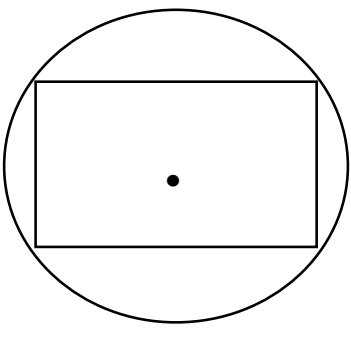

(d)

i

Estos muestran que la noción de estar inscrito no esta clara en los estudiantes y parecería ser una primera dificultad. Después de discutir un poco sobre el significado de estar inscrito se acuerda que la gráfica (d), propuesta por el alumno $\mathrm{P}$, es la traducción correspondiente al enunciado.

Ahora se continúa con el enunciado "... exprese el área del rectángulo como una función de x' y se sugiere la gráfica:

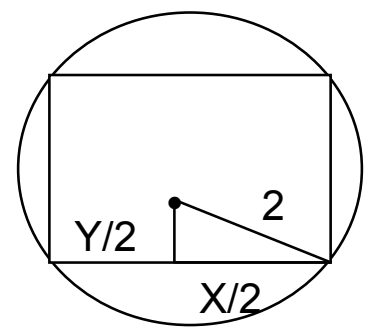

En la iniciación de la búsqueda de la solución (a esta parte del enunciado) se observa que aparecen escrituras como:

$$
A=b . h ; A=b . a ; A=x . h ; A=(b . a) / 2
$$

Nuevamente, se solicita la justificación de cada una de ellas y el alumno K, quien planteó la última expresión aceptó (?) que tenía un error. Puede apreciarse que algunos recuerdan la fórmula de la escuela primaria (variables concretas), otros avanzan un poco en la utilización de la variable (que en este caso está indicada en el enunciado).

Luego de aceptar que se toma como expresión de partida:

$$
A=x \cdot h \text {. }
$$

¿Qué se debe hacer ahora? ¿cómo se sigue?

¿Buscamos quién es $h$ ? y teniendo en cuenta la gráfica se concluye que $h$ es y, en consecuencia se tiene: 


$$
A=x \cdot h=x \cdot y \text { * }
$$

¿Y, ahora qué? se reemplaza y en términos de $x$, ¿Cómo? En la gráfica se tiene como relacionar y con $\mathrm{x}$, aplicando el teorema de Pitágoras. ¿Qué dice el teorema de Pitágoras?

"Hipotenusa al cuadrado es igual a un cateto al cuadrado más el otro cateto al cuadrado y en este caso:

$$
\begin{aligned}
& 2^{2}=\left[\frac{x}{2}\right]+\left[\frac{y}{2}\right] \\
& 4=\frac{x^{2}}{4}+\frac{y^{2}}{4} \\
& 16=x^{2}+y^{2}(i) \\
& 16-x^{2}=y^{2} \\
& \sqrt{ } 16-x^{2}=y \\
& \text { Luego } \quad 4-x=y
\end{aligned}
$$

¿Están de acuerdo con el anterior desarrollo?

Siii

Ante tal exclamación, se presenta:

$$
\begin{gathered}
4=\sqrt{ } 16+\sqrt{26-9}+\sqrt{2} 25-\sqrt{ } 9=5-3=2 \\
\text { Es decir, } 4=2 \\
5=\sqrt{ } 25=\sqrt{ } 16+9=\sqrt{ } 16+\sqrt{ } 9=4+3=7 \\
\text { Luego } 5=7
\end{gathered}
$$

¿Existe un error?

Síii

¿Dónde? ¿En qué paso? Aquíii, en el tercer igual. ¿Por qué?

La raíz de una diferencia no es igual a la diferencia de las raíces o la raíz de una suma no es igual a la suma de las raíces. 
Por lo tanto, no se puede concluir que $y=4-x$, es decir, sólo se tiene que

$$
y=\sqrt{ } 16-x^{2}
$$

Hay alguna restricción o condición?

Si.

$$
x \geq 0 \wedge x \leq 4
$$

\section{¿Porqué?}

Si $X>4$, se tendría para $Y$ un valor imaginario.

¿Puede usted dar una mejor explicación?

Veamos:

$16-X^{2} \geq 0$

$16 \geq X^{2} \geq 0$ Para todo real $X$

$4 \geq|x|$

$-4 \leq x \leq 4$

Como X es la longitud de la base del rectángulo , X no puede ser negativo.

¿Qué, si puede ser? Positivo. ¿Sólo positivo? También puede ser 0.

¿En conclusión? $\quad 0 \leq x \leq 4$

Entonces $y=\sqrt{ } 16-x^{2}, 0 \leq x \leq 4$

Por lo tanto, reemplazando en * se tiene:

$4=x \sqrt{ } 16-x^{2}, \quad 0 \leq x \leq 4$

¿Alguna otra pregunta?

Síii.

¿Cuál?

¿Es posible considerar el siguiente gráfico para encontrar la relación entre $X$ y $Y$ ? Sí. Aplicando Pitágoras en el triángulo rectángulo $A B C$ se tiene:

$$
16=x^{2}+y^{2}
$$




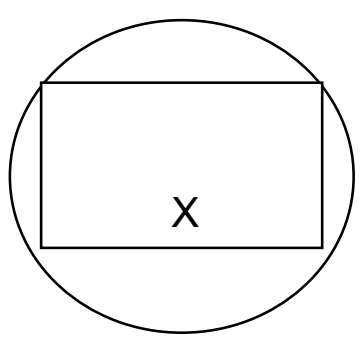

y, estaríamos en el planteamiento (1) del desarrollo anterior.

\section{CONCLUSIONES}

- En una situación como la presentada, muy simple para el profesor, pero muy densa para el alumno se requiere que éste tenga varios conceptos previos que le permitan iniciar con éxito la búsqueda de una solución. Entre ellos están: la fórmula del área para un rectángulo, el teorema de Pitágoras, manejo de ecuaciones y de desigualdades, búsqueda de condiciones para las variables, etc.

- Otro elemento relevante corresponde a las traslaciones de las diferentes representaciones de un enunciado, tales como: de lo verbal a lo gráfico, de lo gráfico a lo simbólico, de lo verbal a lo simbólico, etc. Aquí, se presume que es muy fácil pasar de una representación a otra, tal vez por el poco tiempo disponible y la necesidad de avanzar" en el desarrollo de una temática, pero parecería necesario permitir que cada estudiante haga su traslación y luego se discuta con los compañeros y el profesor para precisar la traslación adecuada.

- A pesar de que un enunciado privilegie una representación, es posible que los alumnos realicen lecturas sobre ella y propongan nuevas interpretaciones, permitiendo con ello dinamizar el proceso que se realiza y obligando al profesor a releer y tal vez a repensar.

-Etc.?

\section{BIBLIOGRAFIA}

1. JANVIER, C. (editor) 1987. Problems of representation in the teaching and learning of mathematics. Londres: LEA Publ. Londres.

2. STEWART, James. Cálculo. México: Grupo Editorial Iberoamericano. 1994. 